\title{
Relación de la ansiedad y la depresión con la asertividad sexual
}

\author{
M. Reina Granados, Pablo Vallejo-Medina y Juan Carlos Sierra \\ Universidad de Granada
}

RESUMEN

Este estudio plantea examinar la relación de la ansiedad y la depresión con la asertividad sexual expresada como inicio y rechazo de la actividad sexual, y prevención del embarazo y enfermedades de transmisión sexual. Una muestra incidental de 632 adultos españoles de distintas edades y situación social (368 hombres y 264 mujeres), contestó a la versión española de la Sexual Asertiveness Scale, al Cuestionario de Ansiedad Estado/Rasgo y al Inventario de Depresión Estado/Rasgo. Los resultados muestran que la asertividad sexual se asocia a los estados de ánimo. Así, en las mujeres, la depresión y la ansiedad explican parte de la varianza de la asertividad sexual, mientras que la depresión predice una parte de la asertividad sexual de rechazo en los varones. Se discute la inclusión de la asertividad sexual y los estados de ánimo de forma conjunta en futuras investigaciones $y$ diagnósticos clínicos.

Palabras clave: asertividad sexual, ansiedad, depresión, salud sexual.

\begin{abstract}
This study proposes to examine the relationship between anxiety and depression with sexual assertiveness expressed as initiation and refusal of sexual activity and sexually transmitted diseases and pregnancy prevention. An incidental sample of 632 Spanish adults of different ages and social status (368 men and 264 women) answered the Spanish version of the Sexual Assertiveness Scale, the State-Trait Anxiety Inventory and the State-Trait Depression Inventory. Results show that sexual assertiveness is associated with mood. In women, depression and anxiety accounts for a portion of the variance in sexual assertiveness, while depression predicts a part of refusal assertiveness in men. It discusses the inclusion for future research and clinical diagnosis sexual assertiveness and moods together.
\end{abstract}

Keywords: sexual assertiveness, anxiety, depression, sexual health. 


\section{Relación de la ansiedad y la depresión con la asertividad sexual}

La asertividad sexual (AS) se define como la habilidad para iniciar la actividad sexual, rechazar la actividad sexual no deseada, así como negociar las conductas sexuales deseadas, el empleo de métodos anticonceptivos y los comportamientos sexuales más saludables (Morokoff et al., 1997). La AS se compone de tres áreas: asertividad de de inicio, asertividad de rechazo, y asertividad en la prevención de embarazo y enfermedades de transmisión sexual (E-ETS). La primera representa la frecuencia que tiene una persona para emprender una relación sexual de forma deseada. La segunda se refiere a la habilidad que tiene una persona en impedir una relación sexual no deseada. Y la AS E-ETS describe la reiteración con la que una persona insiste en el uso de métodos anticonceptivos de barrera de látex con su pareja (Morokoff et al., 1997). Santos-Iglesias y Sierra (2010) señalaron que el estudio de la AS comprende principalmente tres aspectos de la salud sexual: funcionamiento sexual, victimización sexual y conductas sexuales de riesgo.

La AS de inicio se vincula con el funcionamiento sexual, el cual incluye el deseo, la excitación, el orgasmo y satisfacción (Carrobles \& Sanz, 1991). La AS de inicio ha sido asociada principalmente con la satisfacción, ya que cuanto mayor sea la capacidad de la persona para expresar sus preferencias en su propia experiencia y actividad sexual (Morokoff et al., 1997), mayor será la satisfacción experimentada (Greene \& Faulkner, 2005; Haavio-Mannila \& Kontula, 1997; Hurlbert, 1991). El deseo, del mismo modo, fue relacionado en sentido posi- tivo por Hurlbert (1991) con la AS de inicio. Por su parte, Apt, Hurlbert y Powell (1993) señalaron que la diferencia entre la AS del hombre y la AS de la mujer predijo mayor deseo sexual en el varón que en la mujer. La AS de inicio ha sido considerada como predictor de la excitación, existiendo una relación positiva entre ambas (Althof, Perelman \& Rosen, 2011; Hurlbert, Apt \& Rabhel, 1993). El último aspecto a destacar del funcionamiento sexual unido a este tipo de AS es el orgasmo: las mujeres con elevada AS de inicio indican mayor consistencia y número de orgasmos (Hurlbert, 1991; Hurlbert, Apt et al., 1993; Hurlbert, White, Powell \& Apt, 1993; Walker, 2006). La AS de rechazo se asocia con la victimización sexual. Mujeres víctimas de este tipo de abusos manifiestan menor AS de rechazo (Katz, May, Sörensen \& DelTosta, 2010; Kearns \& Calhoun, 2010; Livingston, Testa \& VanZile-Tamsen, 2007; Morokoff et al., 1997). Más aún, la baja AS de rechazo ha sido considerada como predictor de la victimización sexual por Livingston et al. (2007) e intervino como mediador entre la resistencia o sumisión ante una agresión de esta clase (VanZile-Tamsen, Testa \& Livingston, 2005). La AS E-ETS compete a las conductas sexuales de riesgo: a mayor nivel de AS E-ETS menor tendencia a involucrarse en actividades sexuales de riesgo (Noar, Morokoff \& Redding, 2002). El uso del preservativo es considerado como un aspecto elemental en este caso, pues es contraceptivo y profiláctico de las ETS. Además, según Yestmont (1992), la AS E-ETS predice de forma positiva el uso del preservativo, siendo este hecho confirmado en investigaciones posteriores (Bartlett \& Shelton, 2010; Morokoff et al., 2009; Weinstein, Walsh \& 
Ward, 2008). Por consiguiente, la AS ha sido relacionada positivamente con la contracepción (Weinstein et al., 2008) y con el aumento en la evitación ante el Virus de la Inmunodeficiencia Humana (VIH) (Morokoff et al., 1997; Onuoha \& Munakata, 2005). Siguiendo esta línea, la intervención en prevenir las ETS refuerza la AS en mujeres y hombres según confirmaron los estudios de Bartlett y Shelton (2010), Bertens, Eiling, Van den Borne y Schaalma (2009), Di Noia y Schinke (2007), Kelly, Lawrence, Hood y Brasfield (1989) y Sikkema, Winett y Lombard (1995), entre otros.

Queda, por tanto, demostrado que la AS se asocia al funcionamiento sexual, la victimización sexual y las conductas sexuales de riesgo. Por otra parte, estas tres áreas también se han vinculado a los estados de ánimo (EA). Zubeidat y Sierra (2003) analizaron la influencia de la ansiedad y la depresión sobre el deseo sexual. Según estos autores, ambas afectan de modo negativo al deseo sexual. Siguiendo esta misma línea, mujeres diagnosticadas con depresión puntuaron bajo en deseo sexual, mientras que mujeres sin este diagnóstico presentaban un mayor deseo sexual (Cyranowski et al., 2004). Tanto en hombres como en mujeres, la excitación sexual disminuye por la presencia de EA negativos (ansiedad y depresión) (Bancroft et al., 2003; Lutfey, Link, Rosen, Wiegel \& McKinlay, 2009). En cuanto al orgasmo, Lutfey et al. (2009) asociaron la depresión a menor excitación y orgasmos. McCabe y Dalaney (1992), en una revisión sobre la anorgasmia en mujeres, informaron de la correlación significativa entre ésta y la ansiedad. La satisfacción sexual se ve afectada por el bienestar psicológico y por la ansiedad, influyendo el primero de manera positiva y la segunda de forma negativa (Carrobles, Gámez-Guadix \& Almendros, 2011).

Los resultados de Carr y Szymanski (2011) indicaron que la victimización sexual en mujeres se relaciona positivamente con la depresión. Además, el hecho de padecer victimización por parte de la pareja ha sido asociado con depresión (Macy, Nurius \& Norris, 2006; Morokoff et al., 2009; Plotzker, Metzger \& Holmes, 2007). Kimerling, Álvarez, Pavao, Kaminski y Baumrind (2007) vincularon la victimización no solo con la depresión sino también con la ansiedad: mujeres que habían sufrido un episodio de victimización sexual eran más propensas a padecerla. Es más, la depresión y la ansiedad, siendo secuelas de la victimización sexual (Finkelhor, Ormrod \& Turner, 2007), pueden actuar como precipitantes de una victimización sexual futura (Cuevas, Finkelhor, Clifford, Ormrod, \& Turner, 2010; Zurbriggen, Gobin, \& Freyd, 2010).

Por su parte, la depresión y las conductas sexuales de riesgo se encuentran relacionadas, es decir, a mayores niveles de depresión más conductas de riesgo son cometidas, siendo los hombres con depresión más arriesgados en contactos sexuales que las mujeres (Lehrer, Shrier, Gortmaker \& Buka, 2006; Morokoff et al., 2009). Igualmente, estudios sobre la ansiedad y las conductas sexuales de riesgo revelaron conclusiones similares: el aumento de la ansiedad condujo a un mayor número de estos comportamientos (Bancroft, Carnes \& Janssen, 2005; Elkington, Bauermeister \& Zimmerman, 2010). 
Cabe señalar que Morokoff et al. (2009) relacionaron la AS con la depresión, de modo que bajos niveles de AS de uso del preservativo aparecían asociados a altos niveles de depresión. Se demostró que la reducción de los niveles de depresión incrementaba la AS E-ETS (Morokoff et al., 2009; Rickert, Neal, Wiemann \& Berenson, 2000). De ahí que, sea razonable pensar que la AS en sus tres vertientes (inicio, rechazo y prevención de embarazo-ETS) esté vinculada de alguna manera con la ansiedad y la depresión) (véase la Figura 1).
Kaltiala-Heino \& Björkqvist, 2011; Kashdan et al., 2011; Morokoff et al., 2009).

\section{Método}

\section{Participantes}

La muestra estaba compuesta por 632 adultos españoles de distintas edades y situación social (368 hombres y 264 mujeres). Las edades de las mujeres estaban comprendidas entre 18 y 51 años $(M=25,93, D T=7,55)$ y las de los varones entre 18 y 66 años $(M=35,21$, $D T=11,59)$, siendo las diferencias estadísti-

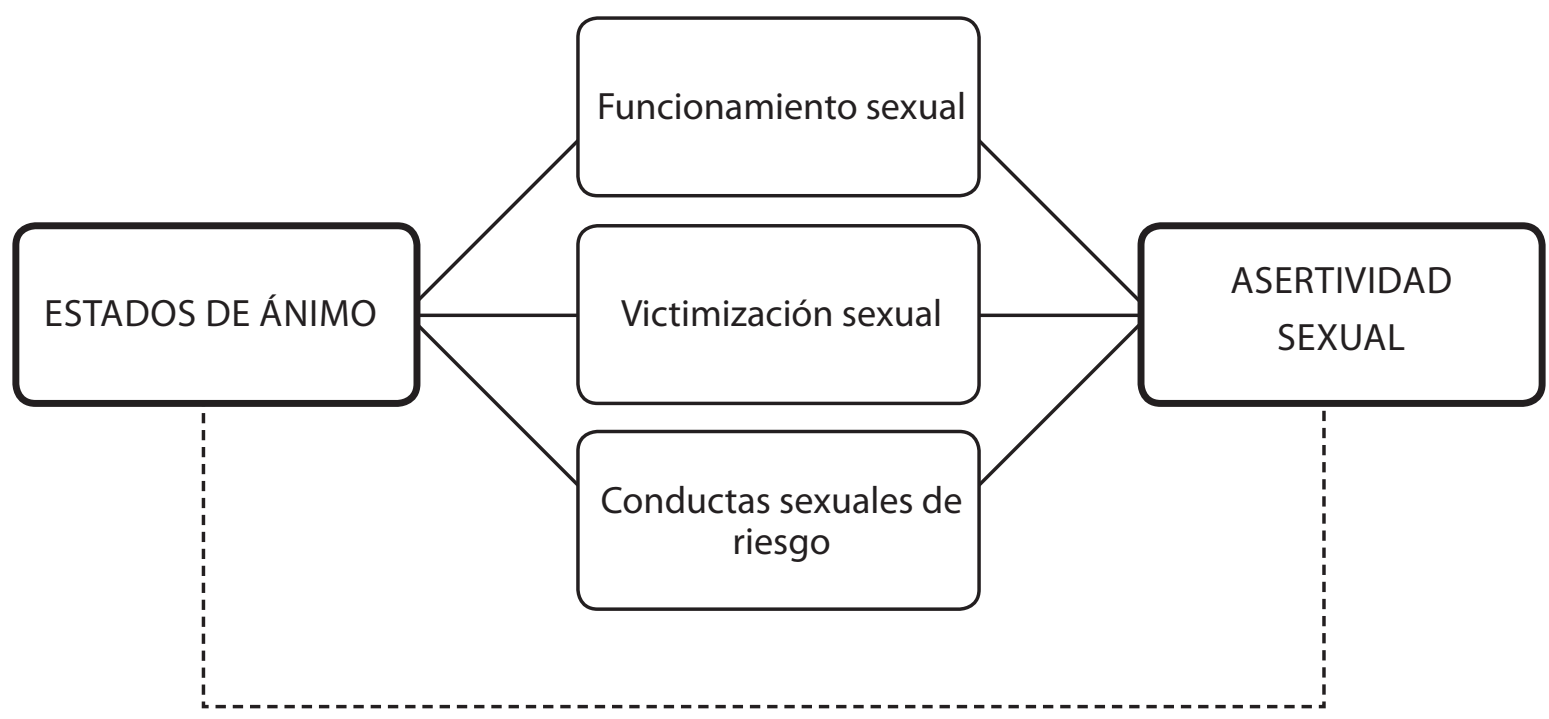

Figura 1. Posible relación entre los estados de ánimo y la asertividad sexual.

Por tanto, el objetivo del presente estudio descriptivo transversal de poblaciones mediante muestras no probabilísticas (Montero \& León, 2007) es examinar la relación de la ansiedad y la depresión con la AS de inicio, de rechazo y E-ETS. Ello lleva a plantear la siguiente hipótesis: se encontrará una correlación negativa entre los niveles de ansiedad y depresión, y la AS de inicio, rechazo y E-ETS (Hankin, Mermelstein \& Roesch, 2007; Hyde, Mezulis \& Abramson, 2008; Isomaa, Isomaa, Marttunen, camente significativas $t(621,90)=12,01, p=$ 0,01 . Las características sociodemográficas de la muestra se pueden observar en la Tabla 1. Existen diferencias significativas en el tipo de relación de los sujetos en función del sexo $X^{2}(5)=63,03, p<0,01$. De igual manera hay diferencias significativas en el nivel académico de los sujetos respecto al sexo $X^{2}(4)=98,60, p$ $<0,01$. Todos los participantes se seleccionaron mediante un muestreo no probabilístico de tipo incidental. 
Tabla 1

Descripción de la muestra

\begin{tabular}{llrr}
\hline \multirow{2}{*}{ Variables } & & \multicolumn{2}{c}{ Sexo } \\
& & Mujeres & Hombres \\
\hline \multirow{3}{*}{ Tipo de pareja } & Sin pareja & $27,10 \%$ & $22,10 \%$ \\
& Pareja menos de 6 meses & $12,60 \%$ & $6,60 \%$ \\
& Pareja más de 6 meses & $46,20 \%$ & $28,10 \%$ \\
& Casados & $11,80 \%$ & $39,30 \%$ \\
& Separados & $2,30 \%$ & $3,30 \%$ \\
Nivel de formación & Viudos & $0,0 \%$ & $0,50 \%$ \\
& Sin estudios & $0,40 \%$ & $1,40 \%$ \\
& Secundaria & $2,70 \%$ & $9,30 \%$ \\
Relaciones sexuales en & $10,40 \%$ & $35,40 \%$ \\
el último mes & Ciclo de Grado Superior & $13,50 \%$ & $20,30 \%$ \\
\hline
\end{tabular}

\section{Instrumentos}

Sexual Assertiveness Scale (SAS; Morokoff et al., 1997). Se utilizó la versión española del Sexual Asertiveness Scale (SAS; Morokoff et al., 1997) de Sierra, Vallejo-Medina y Santos-Iglesias (2011). Esta escala contestada en formato tipo Likert entre 0 (nunca) y 4 (siempre) está compuesta por 18 ítems que recogen las tres dimensiones de la AS: Inicio $(\omega=0,80)$, Rechazo $(\omega=0,76)$ y Prevención de Embarazo y Enfermedades de Transmisión Sexual $(\omega=0,85)$. Puntuaciones elevadas indican mayor AS. Al igual que en la validación española, en la muestra de este estudio también se obtuvieron coeficientes de fiabilidad adecuados: 0,78 para Inicio, 0,72 para Rechazo y 0,86 para $E-$ ETS en mujeres; mientras, en hombres los índices fueron de $0,65,0,73$ y 0,82 , respectivamente. El cuestionario ha demostrado ser equivalente entre hombres y mujeres Sierra, Santos-Iglesias y Vallejo-Medina (2012).
Cuestionario de Ansiedad Estado/Rasgo (STAl; Spielberger, Gorsuch \& Lushene, 1986). Se trata de un cuestionario con escala de respuesta tipo Likert de 40 ítems que evalúa la ansiedad como estado (condición emocional transitoria) y como rasgo (propensión ansiosa relativamente estable). El coeficiente de alfa de Cronbach para Ansiedad estado es 0,92 y para Ansiedad rasgo 0,87. En el presente estudio se obtuvieron para mujeres coeficientes de 0,92 (Ansiedad estado) y 0,91 (Ansiedad rasgo), mientras que para hombres 0,80 (Ansiedad estado) y 0,76 (Ansiedad rasgo). En un estudio reciente Guillén-Riquelme y Buela-Casal (2011) revisan las propiedades psicométricas del STAI e informan resultados óptimos.

Inventario de Depresión Estado/Rasgo (IDER; Spielberger, Buela-Casal \& Agudelo, 2008). Constituye un autoinforme de 20 ítems que evalúan el grado de afectación o estado y 
la frecuencia de ocurrencia o rasgo que el sujeto muestra en relación con los componentes afectivos de la depresión. Las opciones de respuesta son tipo Likert desde 1 (nada) hasta 4 (mucho). El coeficiente de alfa de Cronbach de Depresión estado para mujeres es 0,72 y 0,60 para los hombres, mientras que Depresión rasgo presenta en mujeres una fiabilidad de 0,78 y 0,64 en hombres. En la muestra de mujeres de este estudio se obtuvieron valores de 0,90 para Depresión estado y 0,88 para Depresión rasgo; en la de hombres se obtuvieron valores de 0,91 para Depresión estado y de 0,88 para Depresión rasgo.

\section{Procedimiento}

Dos evaluadores llevaron a cabo la recogida de datos una vez instruidos específicamente para la aplicación de la metodología de este estudio. La evaluación se llevó a cabo en diferentes centros e instituciones concurridos de personas mayores de edad. Mediante un cuadernillo de respuestas autoadministrado los participantes, de forma voluntaria, contestaron a las preguntas bajo las premisas de escrupuloso respeto en la protección de datos y anonimato de los encuestados.

\section{Resultados}

En primer lugar se comprobó si existían diferencias entre hombres y mujeres en asertividad sexual, difiriendo ambos en AS de inicio $t(492,79)=-7,01, p<0,001)$, AS de rechazo $t(500,14)=-16,25, p<0,001)$ y AS E-ETS $t(390,20)=26,75, p<0,001)$. Debido a las diferencias en AS por sexo y a las diferencias sociodemográficas encontradas entre hombres y mujeres, se procedió a realizar los análisis de forma separada para ambas muestras.

En la Tabla 2 se observa la matriz de correlaciones parciales de Pearson (controlando la edad) de las tres dimensiones de la AS con la ansiedad y depresión, como rasgo y como estado, tanto para hombres como para mujeres.

A continuación se llevó a cabo una regresión lineal simple para estimar qué dimensiones de la ansiedad y de la depresión predicen cada una de las modalidades de la AS. Para controlar la posible influencia que la edad tiene sobre la regresión se ha procedido a calcu-

Tabla 2

Correlaciones de la ansiedad y depresión con la asertividad sexual controlando la edad

\begin{tabular}{llccc}
\hline Sexo & Estados de Ánimo & AS Inicio & AS Rechazo & AS E-ETS \\
\hline \multirow{4}{*}{ Mujeres } & Ansiedad-Estado & $-0,20^{* *}$ & $-0,24^{* *}$ & $-0,17^{* *}$ \\
& Ansiedad-Rasgo & $-0,25^{* *}$ & $-0,28^{* *}$ & $-0,18^{* *}$ \\
& Depresión-Estado & $-0,33^{* *}$ & $-0,19^{* *}$ & $-0,15^{*}$ \\
& Depresión-Rasgo & $-0,32^{* *}$ & $-0,29^{* *}$ & $-0,13^{*}$ \\
& Ansiedad-Estado & $-0,02$ & 0,08 & $-0,07$ \\
& Ansiedad-Rasgo & $-0,02$ & $0,16^{* *}$ & 0,01 \\
& Darones & $-0,06$ & $0,22^{* *}$ & 0,02 \\
& Depresión-Estado & $-0,09$ & $0,15^{* *}$ & $-0,01$ \\
\hline
\end{tabular}

${ }^{* *} p<0,01 ;{ }^{*} p<0,05$. 
lar la interacción entre las variables independientes y la edad. Para ello se ha calculado el producto de la edad por las puntuaciones $Z$ de cada variable independiente en cuestión. A continuación, se realizó una regresión jerárquica, en la que en un primer paso se incluía exclusivamente la variable independiente y en el segundo paso se incluía la interacción de la edad con la independiente tipificada. De este modo, si el segundo paso no resulta significativo, se asume que la edad no está influyendo en la recta de regresión. Para las mujeres no se halló un efecto significativo de la edad sobre los efectos que ansiedad y depresión producen sobre la AS (influencia de la edad sobre el efecto de los EA en la AS de inicio $p=0,41$ y correlación parcial $=-0,05$; influencia de la edad sobre el efecto de los EA en la AS de rechazo $p$ $=0,60$ y correlación parcial $=0,03$; influencia de la edad sobre el efecto de los EA en la AS de E-ETS $p=0,88$ y correlación parcial $=0,01$ ). En los hombres tampoco se ha encontrado una influencia de la edad sobre la ecuación de predicción del rechazo (influencia de la edad sobre el efecto de los EA en la AS de rechazo $p$ $=0,22$ y correlación parcial $=0,06$ ). Por tanto, se procedió a realizar la regresión lineal simple para mujeres y hombres. En la muestra de mujeres se comprueba que depresión estado explica el $10 \%$ de la AS de inicio ( $R^{2}$ corregida $=0,10, \beta=-0,32, p<0,01)$, depresión rasgo el $8 \%$ de la AS de rechazo ( $R^{2}$ corregida $=0,08, \beta$ $=-0,29, p<0,01)$ y ansiedad rasgo un $3 \%$ de la AS E-ETS ( $R^{2}$ corregida $=0,03, \beta=-0,17, p<$ $0,01)$, existiendo entre todas ellas una relación significativa y negativa. En cuanto a los varones, ninguna dimensión de la ansiedad y de la depresión predice la AS de inicio. Depresión estado explica un $5 \%$ de la AS de rechazo, siendo la relación entre estas dos variables positiva ( $R^{2}$ corregida $\left.=0,05, \beta=0,23, p<0,01\right)$.

Por último, se realizó una comparación de la AS entre grupos niveles bajos y altos de de ansiedad y depresión. Los niveles altos corresponden a puntuaciones en los cuestionarios de ansiedad y depresión por encima del percentil 75 según los baremos de dichos autoinformes. Los niveles bajos pertenecen a valores inferiores al percentil 25 de los mencionados baremos. Tal como se muestra en la Tabla 3, en las mujeres existen diferencias estadísticamente significativas en AS entre los niveles alto y bajo, tanto de ansiedad como de depresión. En varones, como se muestra en la Tabla 4, únicamente existen diferencias significativas en AS de rechazo en función de la depresión estado.

\section{Discusión}

El presente estudio se ha centrado en la relación de la ansiedad y la depresión con las tres dimensiones de la AS (inicio, rechazo y EETS). Cabe señalar que existen diferencias en la magnitud de las correlaciones. En el caso de las mujeres, la ansiedad y depresión (tanto estado como rasgo) correlacionaron con la AS. Se halla una asociación media entre la AS de inicio y la depresión estado y la depresión rasgo. En cambio, la AS de inicio no presenta ningún tipo de relación con ansiedad y depresión en los hombres. En éstos, ansiedad rasgo, depresión estado y depresión rasgo solo correlacionan con la AS de rechazo de forma baja.

Los resultados de la regresión lineal verifican la relación existente entre AS y ansiedad/depre- 
Tabla 3

Asertividad sexual según los niveles de ansiedad y depresión en la muestra de mujeres

\begin{tabular}{|c|c|c|c|c|c|c|c|}
\hline Asertividad sexual & & M & $D T$ & $t$ & $p$ & gl & $d$ Cohen \\
\hline \multirow{8}{*}{ Inicio } & Alta ansiedad estado & 11,94 & 5,35 & \multirow{2}{*}{$-2,75$} & \multirow{2}{*}{0,01} & \multirow{2}{*}{147} & \multirow{2}{*}{0,53} \\
\hline & Baja ansiedad estado & 14,63 & 4,92 & & & & \\
\hline & Alta ansiedad rasgo & 11,86 & 4,90 & \multirow{2}{*}{$-2,84$} & \multirow{2}{*}{0,01} & \multirow{2}{*}{152} & \multirow{2}{*}{0,58} \\
\hline & Baja ansiedad rasgo & 14,70 & 4,82 & & & & \\
\hline & Alta depresión estado & 12,23 & 4,63 & \multirow{2}{*}{$-4,63$} & \multirow{2}{*}{0,00} & \multirow{2}{*}{181} & \multirow{2}{*}{0,72} \\
\hline & Baja depresión estado & 15,50 & 4,49 & & & & \\
\hline & Alta depresión rasgo & 11,85 & 5,18 & \multirow{2}{*}{$-4,69$} & \multirow{2}{*}{0,00} & \multirow{2}{*}{117} & \multirow{2}{*}{0,87} \\
\hline & Baja depresión rasgo & 15,96 & 4,16 & & & & \\
\hline \multirow{8}{*}{ Rechazo } & Alta ansiedad estado & 15,09 & 5,52 & \multirow{2}{*}{$-2,81$} & \multirow{2}{*}{0,01} & \multirow{2}{*}{147} & \multirow{2}{*}{0,52} \\
\hline & Baja ansiedad estado & 17,74 & 4,62 & & & & \\
\hline & Alta ansiedad rasgo & 14,76 & 5,00 & \multirow{2}{*}{$-3,41$} & \multirow{2}{*}{0,00} & \multirow{2}{*}{152} & \multirow{2}{*}{0,68} \\
\hline & Baja ansiedad rasgo & 17,96 & 4,45 & & & & \\
\hline & Alta depresión estado & 14,73 & 4,68 & \multirow{2}{*}{$-3,94$} & \multirow{2}{*}{0,00} & \multirow{2}{*}{181} & \multirow{2}{*}{0,62} \\
\hline & Baja depresión estado & 17,67 & 4,84 & & & & \\
\hline & Alta depresión rasgo & 13,63 & 4,29 & \multirow{2}{*}{$-4,88$} & \multirow{2}{*}{0,00} & \multirow{2}{*}{117} & \\
\hline & Baja depresión rasgo & 17,94 & 4,71 & & & & נט, \\
\hline & Alta ansiedad estado & 15,91 & 5,79 & & & & \\
\hline & Baja ansiedad estado & 19,52 & 5,39 & נכ, &, 0 & J & \\
\hline & Alta ansiedad rasgo & 16,04 & 6,87 & 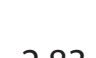 & & & \\
\hline & Baja ansiedad rasgo & 19,50 & 5,60 & - & I & טסו & כד, \\
\hline & Alta depresión estado & 16,85 & 6,35 & & & & \\
\hline & Baja depresión estado & 19,23 & 5,56 & $-2,00$ & I & 179 & 0,40 \\
\hline & Alta depresión rasgo & 16,63 & 5,97 & & & & \\
\hline & Baja depresión rasgo & 18,95 & 5,74 & $-2,0 J$ & 0,04 & כוד & 0,40 \\
\hline
\end{tabular}

sión. Tanto en hombres como en mujeres, no parece observarse una influencia de la edad sobre la implicación que los AE tienen sobre la AS. Es de destacar que en las mujeres la AS de inicio se ve afectada por la depresión estado. Asimismo, en cierta medida se estima que la ansiedad rasgo perjudica a la AS de rechazo. Además, la
AS E-ETS se ve afectada de forma negativa por el incremento de la ansiedad rasgo. Por otro lado, en los varones la depresión estado repercute de forma positiva sobre la AS de rechazo. Los resultados encontrados en la muestra de mujeres indican claramente que tanto la ansiedad como la depresión presentan una relación 
Tabla 4

Asertividad sexual según niveles de ansiedad y depresión en la muestra de hombres

\begin{tabular}{|c|c|c|c|c|c|c|c|}
\hline Asertividad sexual & & $M$ & $D T$ & $t$ & $p$ & $g l$ & $d$ Cohen \\
\hline \multirow{8}{*}{ Inicio } & Alta ansiedad estado & 13,99 & 4,56 & \multirow{2}{*}{0,56} & \multirow{2}{*}{0,58} & \multirow{2}{*}{144} & \multirow{2}{*}{0,09} \\
\hline & Baja ansiedad estado & 13,58 & 4,17 & & & & \\
\hline & Alta ansiedad rasgo & 13,87 & 4,33 & \multirow{2}{*}{$-0,15$} & \multirow{2}{*}{0,88} & \multirow{2}{*}{226} & \multirow{2}{*}{0,02} \\
\hline & Baja ansiedad rasgo & 13,97 & 4,24 & & & & \\
\hline & Alta depresión estado & 13,73 & 4,90 & \multirow{2}{*}{$-0,44$} & \multirow{2}{*}{0,66} & \multirow{2}{*}{137} & \multirow{2}{*}{0,07} \\
\hline & Baja depresión estado & 14,05 & 3,65 & & & & \\
\hline & Alta depresión rasgo & 13,44 & 4,50 & \multirow{2}{*}{$-0,94$} & \multirow{2}{*}{0,35} & \multirow{2}{*}{167} & \multirow{2}{*}{0,15} \\
\hline & Baja depresión rasgo & 14,08 & 4,01 & & & & \\
\hline \multirow{8}{*}{ Rechazo } & Alta ansiedad estado & 11,24 & 5,22 & \multirow{2}{*}{0,10} & \multirow{2}{*}{0,92} & \multirow{2}{*}{144} & \multirow{2}{*}{0,02} \\
\hline & Baja ansiedad estado & 11,15 & 5,68 & & & & \\
\hline & Alta ansiedad rasgo & 11,97 & 5,25 & \multirow{2}{*}{1,49} & \multirow{2}{*}{0,14} & \multirow{2}{*}{226} & \multirow{2}{*}{0,22} \\
\hline & Baja ansiedad rasgo & 10,80 & 5,64 & & & & \\
\hline & Alta depresión estado & 12,86 & 5,48 & \multirow{2}{*}{2,60} & \multirow{2}{*}{0,01} & \multirow{2}{*}{137} & \multirow{2}{*}{0,45} \\
\hline & Baja depresión estado & 10,39 & 5,62 & & & & \\
\hline & Alta depresión rasgo & 12,61 & 4,85 & \multirow{2}{*}{2,50} & \multirow{2}{*}{0,13} & \multirow{2}{*}{167} & ר17 \\
\hline & Baja depresión rasgo & 10,40 & 5,71 & & & & 0,42 \\
\hline & Alta ansiedad estado & 13,51 & 6,45 & 160 & 010 & 11 & ק \\
\hline & Baja ansiedad estado & 15,36 & 6,87 & & & & \\
\hline & Alta ansiedad rasgo & 13,42 & 6,37 & & & & \\
\hline & Baja ansiedad rasgo & 14,36 & 7,42 & $-0,0>$ & 0,30 & STl, & 0,14 \\
\hline & Alta depresión estado & 13,86 & 7,00 & & & & \\
\hline & Baja depresión estado & 12,75 & 6,85 & (4), & נכ, & 150 & 0,10 \\
\hline & Alta depresión rasgo & 14,04 & 7,04 & & & & \\
\hline & Baja depresión rasgo & 13,29 & 6,86 & 0,00 & וכ, & 100 & 0,00 \\
\hline
\end{tabular}

negativa con la AS. Por el contrario, en los varones los resultados son importantes por no haber sido estudiados con anterioridad y porque la relación es positiva entre las variables medidas, es decir, la asertividad de rechazo aumenta al hacerlo la depresión estado. Según Sigmon et al. (2007) el género tiene un papel importante en el curso de numerosos trastornos psicológicos. En los adultos, la depresión y la ansiedad es mayor en mujeres que en hombres como mostraron los estudios de Hankin et al. (2007), Hyde et al. (2008), Isomaa et al. (2011), Kashdan et al. (2011) y Morokoff et al. (2009), entre otros. Otras afecciones como el estrés también son más pa- 
decidas por las mujeres que por los hombres (Hankin et al., 2007; Olff, Langeland, Draijer \& Gersons, 2007). Santos-Iglesias, Sierra y Vallejo-Medina (en prensa) señalan que la AS está determinada por diferentes variables (funcionamiento sexual, actitudes sexuales o abusos en la pareja), pero éstas juegan un papel diferente en hombres y mujeres. Esta diferencia entre ambos sexos puede ser debida a normas de género como la pasividad sexual de las mujeres y la subordinación de los intereses sexuales de éstas a los de ellos (Bay-Cheng \& Eliseo-Arras, 2008). Así mismo, según Kiefer y Sanchez (2007), los hombres son educados socialmente para iniciar y dirigir las actividades sexuales con mujeres. Según Zubeidat y Sierra (2003), la depresión ejerce un efecto negativo sobre el deseo sexual, lo que podría explicar que en los hombres la AS de rechazo aumente al hacerlo la depresión.

Las mujeres con altas puntuaciones en depresión o en ansiedad tienen un nivel bajo en cualquier tipo de AS. Esto se aprecia también al comparar esos resultados con el baremo del SAS para la población general de mujeres españolas (Sierra et al., 2012). Todas las mujeres con elevada ansiedad y depresión se encuentran entre el percentil 25 y 35 del mencionado baremo, lo que pone de manifiesto la relación que se viene discutiendo. Del mismo modo, en la muestra de hombres se observa la relación positiva que existe entre la AS de rechazo y la depresión estado. Al comparar con el mencionado baremo del SAS para hombres españoles, la AS de rechazo está situada en el percentil 65 (por encima de la media) en hombres con elevados niveles de depresión.
Con los resultados obtenidos se reconoce que hay una reciprocidad entre la AS y los EA. Las implicaciones con la salud sexual son evidentes. Sierra, Santos, Gutiérrez-Quintanilla, Gómez y Maeso (2008) exponen que la AS constituye un factor determinante de la salud sexual. En consecuencia, los programas de prevención e intervención para un adecuado funcionamiento sexual, victimización sexual y conductas sexuales de riesgo deberían examinar el estado emocional de los individuos, teniendo en cuenta las diferencias por sexo evidentes que muestra este estudio en cuanto a la relación de ambos constructos. Dichos programas incluyen a la AS como una variable a potenciar (Bartlett \& Shelton, 2010; Bertens et al., 2009; Di Noia \& Schinke, 2007), pues es beneficiosa para las tres dimensiones citadas de la salud sexual (Jacobs \& Thomlison, 2009; Saleh-Onoya et al., 2008; Štulhofer, Graham, Božičević, Kufrin \& Ajduković, 2009).

Por último, se deben señalar algunas limitaciones del estudio. Una de ellas está asociada con la generalización de los resultados. Al tratarse de un muestreo de tipo no probabilístico no es posible extrapolar los resultados a poblaciones mayores. Por ello, se recomienda que en futuras investigaciones se realice un muestreo de tipo probabilístico. Otra limitación se refiere al hecho de no poder comparar entre las muestras de ambos sexos, aspecto que sería interesante tratar ya que se ha podido observar que esas diferencias son potenciales. No obstante, esta circunstancia lleva a recomendar que se elabore una investigación comparativa entre hombres y mujeres, debido al valor de la información que se podría conseguir con el 
análisis de la variable sexo. Por otra parte, una propuesta para investigaciones futuras sería la de ampliar el estudio a diferentes poblaciones, con el objetivo de realizar comparaciones en cuanto a diferencias entre sexo, muestra clínica, otros ámbitos contextuales y temporales, ya que las características de cada muestra pueden plantear nuevas cuestiones relacionadas con las variables de estudio, como otros factores desencadenantes que puedan influir en ellas. Cualquier factor, como es el caso del estado emocional, que interfiera en la AS repercute en la salud sexual. Otro punto a tener en cuenta en posteriores trabajos y en estudios clínicos es considerar la AS, la depresión y ansiedad de manera conjunta con el fin de completar aspectos obviados con anterioridad.

\section{Referencias}

Althof, S. E., Perelman, M. A. \& Rosen, R.C. (2011). The subjective sexual arousal scale for men (SSASM): Preliminary development and psychometric validation of a multidimensional measure of subjective male sexual arousal. Journal of Sexual Medicine, 8, 2255-2268. doi:10.1111/j.1743-6109.2011.02319.x

Apt, C., Hurlbert, D. F. \& Powell, R. D. (1993). Men with hypoactive sexual desire: The role of interpersonal dependency and assertiveness. Journal of Sex Education and Therapy, 19, 108-116.

Bancroft, J., Carnes, L. \& Janssen, E. (2005). Unprotected anal intercourse in HIV-positive and HIV-negative gay men: The relevance of sexual arousability, mood, sensation seeking, and erectile problems. Archives of Sexual Behavior, 34, 299-305. doi:10.1007/ s10508-005-3118-6

Bancroft, J., Janssen, E., Strong, D., Carnes, L., Vukadinovic, Z. \& Long, J. S. (2003). The relation between mood and sexuality in hetero- sexual men. Archives of Sexual Behavior, 32, 217-230. doi:10.1023/A:1023409516739

Bartlett, R. \& Shelton, T. (2010). Feasibility and initial efficacy testing of an HIV prevention intervention for black adolescent girls. Issues in Mental Health Nursing, 31, 731-738. doi:10.3109/01612840.2010.505313

Bay-Cheng, L. Y. \& Eliseo-Arras, R. K. (2008). The making of unwanted sex: Gendered and neoliberal norms in college women's unwanted sexual experiences. Journal of Sex Research, 45, 386-397. doi:10.1080/00224490802398381

Bertens, M., Eiling, E. M., Van den Borne, B. \& Schaalma, H. P. (2009). Uma Tori! Evaluation of an STI/HIV-prevention intervention for Afro-Caribbean women in the Netherlands. Patient Education and Counseling, 75, 77-83. doi:10.1016/j.pec.2008.09.002

Carr, E. R. \& Szymanski, D. M. (2011). Sexual objectification and substance abuse in young adult women. Counseling Psychologist, 39, 39-66. doi:10.1177/0011000010378449

Carrobles, J. A., Gámez-Guadix, M. \& Almendros, C. (2011). Funcionamiento sexual, satisfacción sexual y bienestar psicológico y subjetivo en una muestra de mujeres Españolas. Anales de Psicología, 27, 27-34.

Carrobles, J. A. \& Sanz, A. (1991). Terapia sexual. Madrid: Fundación Universidad Empresa.

Cuevas, C. A., Finkelhor, D., Clifford, C., Ormrod, R. K. \& Turner, H. A. (2010). Psychological distress as a risk factor for re-victimization in children. Child Abuse and Neglect, 34, 235-243. doi:10.1016/j.chiabu.2009.07.004

Cyranowski, J. M., Bromberger, J., Youk, A., Matthews, K., Kravitz, H. M. \& Powell, L. H. (2004). Lifetime depression history and sexual function in women at midlife. Archives of Sexual Behavior, 33, 539-548. doi:10.1023/ B:ASEB.0000044738.84813.3b

Di Noia, J. \& Schinke, S. P. (2007). Gender-specific HIV prevention with urban early-adolescent girls: Outcomes of the keepin' it safe 
program. AIDS Education and Prevention, 19, 479-488. doi:10.1521/aeap.2007.19.6.479

Elkington, K. S., Bauermeister, J. A. \& Zimmerman, M. A. (2010). Psychological distress, substance use, and HIV/STI risk behaviors among youth. Journal of Youth and Adolescence, 39, 514-527. doi:10.1007/s10964010-9524-7

Finkelhor, D., Ormrod, R. K. \&Turner, H. A. (2007). Poly-victimization and trauma in a national longitudinal cohort. Development and Psychopathology, 19, 149-166. doi:10.1017/ S0954579407070083

Greene, K. \& Faulkner, S. L. (2005). Gender, belief in the sexual double standard, and sexual talk in heterosexual dating relationships. Sex Roles, 53, 239-251. doi:10.1007/ s11199-005-5682-6

Guillén-Riquelme, A. \& Buela-Casal, G. (2011). Actualización psicométrica y funcionamiento diferencial de los ítems en el State Trait Anxiety Inventory (STAI). Psicothema, 23, 510-515.

Haavio-Mannila, E. \& Kontula, O. (1997). Correlates of increased sexual satisfaction. Archives of Sexual Behavior, 26, 399-419. doi:10.1023/A:1024591318836

Hankin, B. L., Mermelstein, R. \& Roesch, L. (2007). Sex differences in adolescent depression: Stress exposure and reactivity models. Child Development, 78, 279-295. doi:10.1111/j.1467-8624.2007.00997.x

Hurlbert, D. F. (1991). The role of assertiveness in female sexuality: A comparative study between sexually assertive and sexually nonassertive women. Journal of Sex \& Marital Therapy, 17, 183-190. doi:10.1080/00926239108404342

Hurlbert, D. F., Apt, C. \& Rabhel, S. (1993). Key variables to understanding female sexual satisfaction: An examination of women nondistressed marriages. Journal of Sex \& Marital Therapy, 19, 154-165. doi:10.1080/00926239308404899
Hurlbert, D. F., White, L. C., Powell, R. D. \& Apt, C. (1993). Orgasm consistency training in the treatment of women reporting hypoactive sexual desire: An outcome comparison of women-only groups and couplesonly groups. Journal of Behavioral Theory and Experimental Psychiatry, 24, 3-13. doi:10.1016/0005-7916(93)90003-F

Hyde, J. S., Mezulis, A. H. \& Abramson, L. Y. (2008). The ABCs of depression: Integrating affective, biological, and cognitive models to explain the emergence of the gender difference in depression. Psychological Review, 115, 291-313. doi:10.1037/0033295X.115.2.291

Isomaa, R., Isomaa, A. L., Marttunen, M., Kaltiala-Heino, R. \& Björkqvist, K. (2011). Longitudinal concomitants of incorrect weight perception in female and male adolescents. Body Image, 8, 58-63. doi:10.1016/j. bodyim.2010.11.005

Jacobs, R. J. \& Thomlison, B. (2009). Self-silencing and age as risk factors for sexually acquired HIV in midlife and older women. Journal of Aging and Health, 21, 102-128. doi:10.1177/0898264308328646

Kashdan, T. B., Adams, L., Savostyanova, A., Ferssizidis, P., McKnight, P. E. \& Nezlek, J. B. (2011). Effects of social anxiety and depressive symptoms on the frequency and quality of sexual activity: A daily process approach . Behaviour Research and Therapy, 49, 352360. doi:10.1016/j.brat.2011.03.004

Katz, J., May, P., Sörensen, S. \& DelTosta, J. (2010). Sexual revictimization during women's first year of college: Self-blame and sexual refusal assertiveness as possible mechanisms. Journal of Interpersonal Violence, 25, 21132126. doi:10.1177/0886260509354515

Kearns, M. C. \& Calhoun, K. S. (2010). Sexual revictimization and interpersonal effectiveness. Violence and Victims, 25, 504-517. doi:10.1891/0886-6708.25.4.504

Kelly, J. A., Lawrence, J. S., Hood, H. V. \& Brasfield, T. L. (1989). Behavioral intervention to reduce AIDS risk activities. Journal of Con- 
sulting and Clinical Psychology, 57, 60-67. doi:10.1037/0022-006X.57.1.60

Kiefer, A. K. \& Sanchez, D. T. (2007). Men's sexdominance inhibition:Do men automatically refrain from sexually dominant behavior? Personality and Social PsychologyBulletin, 33, 1617-1631. doi:10.1177/014616720730585

Kimerling, R., Alvarez, J., Pavao, J., Kaminski, A. \& Baumrind, N. (2007). Epidemiology and consequences of women's revictimization. Women's Health Issues, 17, 101-106. doi:10.1016/j.whi.2006.12.002

Lehrer, J. A., Shrier, L. A., Gortmaker, S. \& Buka, S. (2006). Depressive symptoms as a longitudinal predictor of sexual risk behaviors among US middle and high school students. Pediatrics, 118, 189-200. doi:10.1542/ peds.2005-1320

Livingston, J. A., Testa, M. \& VanZile-Tamsen, C. (2007). The reciprocal relationship between sexual victimization and sexual assertiveness. Violence Against Women, 13, 298-313. doi:10.1177/107780120629733

Lutfey, K. E., Link, C. L., Rosen, R. C., Wiegel, M. \& McKinlay, J. B. (2009). Prevalence and correlates of sexual activity and function in women: Results from the Boston area community health $(\mathrm{BACH})$ survey. Archives of Sexual Behavior, 38, 514-527. doi:10.1007/ s10508-007-9290-

Macy, R. J., Nurius, P. S. \& Norris, J. (2006). Responding in their best interests: Contextualizing women coping with acquaintance sexual assault. Violence Against Women, 12, 478-500. doi:10.1177/107780120628810

McCabe, M. P. \& Delaney, S. M. (1992). An evaluation of therapeutic programs for the treatment of secondary inorgasmia in women. Archives of Sexual Behavior, 21, 69-89. doi:10.1007/BF01542717

Montero, I. \& León, O. G. (2007). A guide for naming research studies in Psychology. International Journal of Clinical and Health Psychology, 7, 847-862. Recuperado de http://www.aepc.es/ijchp/GNEIP07_es.pdf
Morokoff, P. J., Quina, K., Harlow, L. L., Whitmire, L., Grimley, D. M., Gibson, P. R., ...Burkholder, G. J. (1997). Sexual Assertiveness Scale (SAS) for women: Development and validation. Journal of Personality and Social Psychology, 73, 790-804. doi:10.1037/00223514.73.4.790

Morokoff, P. J., Redding, C. A., Harlow, L. L., Cho, S., Rossi, J. S., Meier, K. S., ... Brown-Peterside, P. (2009). Associations of sexual victimization, depression, and sexual assertiveness with unprotected sex: A test of multifaceted model of HIV risk across gender. Journal of Applied Biobehavioral Research, 14, 30-54. doi:10.1111/j.1751-9861.2009.00039.x

Noar, S. M., Morokoff, P. J. \& Redding, C. A. (2002). Sexual assertiveness in heterosexually active men: A test of three samples. AIDS Education and Prevention, 14, 330-342. doi:10.1521/aeap.14.5.330.23872

Olff, M., Langeland, W., Draijer, N. \& Gersons, B. P. R. (2007). Gender differences in posttraumatic stress disorder. Psychological Bulletin, 133, 183-204. doi:10.1037/00332909.133.2.183

Onuoha, F. N. \& Munakata, T. (2005). Correlates of adolescent assertiveness with HIV avoidance in a four-nation sample. Adolescence, 40, 525-532. Recuperado de http://www. redorbit.com/news/health/281118/correlates_of_adolescent_assertiveness_with_ hiv_avoidance_in_a_four/index.html

Plotzler, R. E., Metzger, D. S. \& Holmes, W. C. (2007). Childhood sexual and physical abuse histories, PTSD, depression, and HIV risk outcomes in women injection drug users: A potential mediating pathway. American Journal of Addiction, 16, 431-438. doi:10.1080/10550490701643161

Rickert, V. I., Neal, W. P., Wiemann, C. M. \& Berenson, A. B. (2000). Prevalence and predictors of low sexual assertiveness. Journal of Pediatric and Adolescent Gynecology, 13, 88-89. doi:10.1016/S1083-3188(00)00016-4 
Saleh-Onoya, D., Braxton, N. D., Sifunda, S., Reddy, P., Ruiter, R., van den Borne, B., ... Wingoo, G. M. (2008). SISTA South Africa: The adaptation of an efficacious HIV prevention trial conducted with African-American women for isiXhosa-speaking South African women. Sahara J, 5, 186-191.

Santos-Iglesias, P. \& Sierra, J. C. (2010). El papel de la asertividad sexual en la sexualidad humana: una revisión sistemática. International Journal of Clinical and Health Psychology, 10, 553-577.

Santos-Iglesias, P., Sierra, J. C. \& Vallejo-Medina, P. (en prensa). Predictors of Sexual Assertiveness: the Role of Sexual Desire, Arousal, Attitudes and Partner abuse. Archives of Sexual Behavior.

Sierra, J. C., Santos, P., Gutiérrez-Quintanilla, J. R., Gómez, P. \& Maeso, M. D. (2008). Un estudio psicométrico del Hurlbert Index of Sexual Assertiveness en mujeres hispanas. Terapia Psicológica, 26, 117-123. doi:10.4067/ S0718-48082008000100010

Sierra, J. C., Santos-Iglesias, P. \& Vallejo-Medina, P. (2012). Evaluación de la equivalencia factorial y métrica de la Sexual Assertiveness Scale (SAS) por sexo y edad. Psicothema, 24, 316-322

Sierra, J. C., Vallejo-Medina, P. \& Santos-Iglesias, P. (2011). Propiedades psicométricas de la versión española de la Sexual Assertiveness Scale (SAS). Anales de Psicología, 27, 17-26.

Sigmon, S. T., Pells, J. J., Edenfield, T. M., Hermann, B. A., Schartel, J. G., LaMattina, S. M.,Boulard, N. E. (2007). Are we there yet? A review of gender comparisons in three behavioral journals through the 20th century. BehaviorTherapy, 38,333-339.doi:10.1016/j. beth.2006.10.003

Sikkema, K. J., Winett, R. A. \& Lombard, D. N. (1995). Development and evaluation of an HIVrisk reduction program for female college students. AIDS Education and Prevention, 7, 145-159.

Spielberger, C. D., Buela-Casal, G. \& Agudelo, D. (2008). IDER, Inventario de Depresión Estado/Rasgo. Madrid:TEA.
Spielberger, C. D., Gorsuch, R. L. \& Lushene, R. E. (1986). Manual for the State-Trait Anxiety Inventory (Self-Evaluation Questionaire). California: Consulting Psychologists Press. Adaptación española. Madrid:TEA.

Štulhofer, A., Graham, C., Božičević, I., Kufrin, K. \& Ajduković, D. (2009). An assessment of HIV/STI vulnerability and related sexual risk-taking in a nationally representative sample of young Croatian adults. Archives of Sexual Behavior, 38, 209-225. doi:10.1007/ s10508-007-9234-8

VanZile-Tamsen, C., Testa, M. \& Livingston, J. A. (2005). The impact of sexual assault history and relationship context on appraisal of and responses to acquaintance sexual assault risk. Journal of Interpersonal Violence, 20, 813-832. doi:10.1177/0886260505276071

Walker, D. P. (2006). Impaired sexual assertiveness and consensual sexual activity as risk factors for sexual coercion in heterosexual college women (Tesis doctoral, Universidad de Miami, Ohio). Recuperado de http:// etd.ohiolink.edu/send-pdf.cgi/Walker\%20 David\%20Pierce.pdf?miami1155324575

Weinstein, R. B., Walsh, J. L. \& Ward, L. M. (2008). Testing a new measure of sexual health knowledge and its connections to students' sex education, communication, confidence, and condom use. International Journal of Sexual Health, 20, 212-221. doi:10.1080/19317610802240279

Yesmont, G. A. (1992). The relationships of assertiveness to college students' safer sex behaviors. Adolescence, 27, 253-272.

Zubeidat, I. \& Sierra, J. C. (2003). Influencia de diferentes factores psicológicos sobre el deseo sexual. Revista Argentina de Clínica Psicológica, 12, 69-83.

Zurbriggen, E. L., Gobin, R. L. \& Freyd, J. J. (2010). Childhood emotional abuse predicts late adolescent sexual aggression perpetration and victimization. Journal of Aggression, Maltreatment and Trauma, 19, 204-223. doi:10.1080/10926770903539631 